Chirurg 2010 $\cdot 81: 1034$

DOI 10.1007/s00104-010-1981-8

Online publiziert: 21. Oktober 2010

(c) Springer-Verlag 2010

\title{
Master of Business Administration als Wundermittel
}

Und dass die Notwendigkeit von Führungskompetenz wesentlicher Erfolgsfaktor für einen Chefarzt ist, ist eine Aussage, mit der sich die Träger von Krankenhäusern, aber auch Institutionen wie die Bundesärztekammer, leider erst in neuerer Zeit auseinandersetzen (Curriculum Führung). Freie und hochschulische Anbieter, aber auch Berufsverbände wie der Bundesverband Deutscher Chirurgen (BDC), sind hier Jahre voraus und haben massiv dazu beigetragen, dass in deutschen Krankenhäusern zunehmend junge Chefärzte agieren, die nicht nur fachlich hochqualifiziert sind, sondern auch gute betriebswirtschaftlich Kenntnisse aufweisen und Mitarbeiter angemessen führen können.

Die Auffassung, dass „persönliche und praktische Eigenschaften in MBA-Programmen nicht gelernt werden können" ist ebenfalls nur eingeschränkt nachvollziehbar. Denn Programme für Ärzte sollen das Studium vor allem Promotor zur Aufdeckung von Schwächen sein und als Stimulans für die Beanspruchung geeigneter Trainings dienen, und zwar lebenslang. Erste Schritte dazu können aber selbstverständlich auch in MBA-Studiengängen gegangen werden.

Was die Kosten und die Dauer anbetrifft: Die Teilnehmergebühren für einschlägige MBA-Programme in Deutschland sind häufig nicht erheblich kostenaufwendiger als diejenigen für „Kurzzeitkurse mit Exklusivcharakter für Chefärzte oder Ärztliche Direktoren". Und was die Zeit anbelangt, ist diese meist auch gut angelegt. Denn die komplexe Einrichtung Krankenhaus ist nicht über zeitlich schmale Fortbildungen zu begreifen. Insbesondere dann nicht, wenn diese ausschließlich die Sicht ärztlicher Funktions- träger oder der Krankenhausleitung widerspiegeln.

Vielmehr muss ein zeitgemäßes Krankenhausmanagement nicht nur angemessen die Patienten-, sondern auch die Mitarbeiterperspektive (und damit auch die Weiterbildungsinteressen der Chefärzte selbst!) berücksichtigen. Eine nachhaltige Vermittlung entsprechender Kenntnisse kann deshalb nicht in Wochenendkursen oder Wochenkursen erfolgen, sondern nur in umfassenderen Programmen. Diese haben auch den Vorteil, dass nicht nur ein Zertifikat, sondern ein berufsqualifizierender Abschluss erworben wird, der den Ärzten auch Perspektiven für andere Berufsfelder verschafft.

Insgesamt betrachtet, ist ein auf krankenhauspezifische Belange orientierter MBA vor allem Ärzten zu empfehlen, die Positionen wie Chefarzt, Ärztlicher Direktor oder Ärztlicher Geschäftsführer anstreben, aber auch Ärzten, die sich den Weg in andere Berufsfelder offen halten wollen.

\section{Korrespondenzadresse \\ Prof. Dr. W. Hellmann \\ Zentrum für Weiterbildung \\ und Technologietransfer (ZWT), \\ Fachhochschule Hannover, \\ Blumhardtstraße 2, 30625 Hannover \\ Hellmann-W@t-online.de}

